\title{
The Design of Angle Auto Direction-adjusting System for Wind Tunnel Motion Mechanism
}

\author{
Xiao Tao $\mathrm{Yu}^{1, \mathrm{a}}$ \\ ${ }^{1}$ AVIC Shenyang Engine Design and Research institute, Shenyang, 110015, China
}

\begin{abstract}
In this article a motion mechanism angle self direction-adjusting controlling system is designed. The main function of this system is to achieve the angle following control with the variation of inflow direction. This article describes the construction and working principle of the angle self direction-adjusting system, aiming to the controlling target the self-adapting fuzzy controller is designed, the controller is then simulated and analyzed after adding square wave signals. It is validated that the self-adapting fuzzy PID controller has a good dynamic, static and robust characteristics.
\end{abstract}

Keywords: angle control; self-adapting; fuzzy control; MATLAB simulation

\section{Introduction}

When the wind tunnel is built up, the performance of the wind tunnel required to be checked out through flow field verification. Flow field verification requires a set of equipment, by fixing probes and movable ones of certain requirements in order to test the parameters of flow stability, dynamic pressure field, flow deviation angle and pressure grads of model at each point on the flow section of testing segment. in the testing process it is required that the probe direction should right face to the inflow direction all the time, which means the motion mechanism need to have angle auto direction-adjusting function. To improve the flow field test efficiency, guarantee the testing accuracy, the motion mechanism angle self direction-adjusting control system is designed $[1,2]$.

\section{Motion mechanism components}

system

The motion mechanism is constructed by a DLK120 beeline anchor cell and two ELK40 single-slide ball screw drive positioning cells as well as $1 \mathrm{D}$ rotary platform and the accessories, the structure is shown in Fig1. There are 4 degrees of freedom for the motion mechanism, namely $\mathrm{X}, \mathrm{Y}, \mathrm{Z}$ and $\mathrm{M}$ axes. Each axis is driven by servomotor, where $M$ axis is angle axis, by driving the $M$ axis moving through motor it is able to achieve probe auto direction-adjusting.

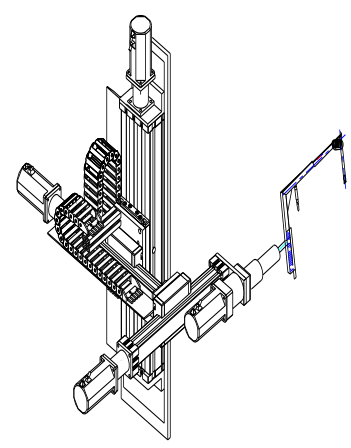

Fig.1. Self-adapting fuzzy PID controller

\section{Working principle}

The basic principle of the motion mechanism angle following system is to transform the pressure signal received by the controlled probes into standard signal through sensors. The signal is compared with real-time adjusting data collected by PLC, and then the deviation signal is sent into servo driver to operate reversible motor rotating in contrary direction; to control the overshoot of the system, $\mathrm{Ka}$, the correction factor, is calculated out through the relative PLC measuring parameters to control the command impulse of the servo driver to make the probe taken by the servomotor rotate to the direction of smaller deviation voltage, in this way the probe is made to achieve self equilibrating in the flow direction.

\section{The design and simulation for the controller of the angle control system}

The self-adapting fuzzy PID controller structure is shown in Fig.2. The core of the self-adapting fuzzy PID controller is to apply PID controller to control system. The inputs of the fuzzy deduction is deviation and deviation varying rate ec, after fuzzy deduction three parameters of PID controller, namely 
$K_{p}, K_{i}, K_{d}$ are auto modified in the parameter modification stage to satisfy the system requirement. It can be seen that since the fuzzy deduction and parameter modification stages are added, even with different control system a good control result is able to be achieved by online setting modifying parameters, meanwhile by using this control method not only the controlled object has a good dynamical performance but also has a good static performance[3].

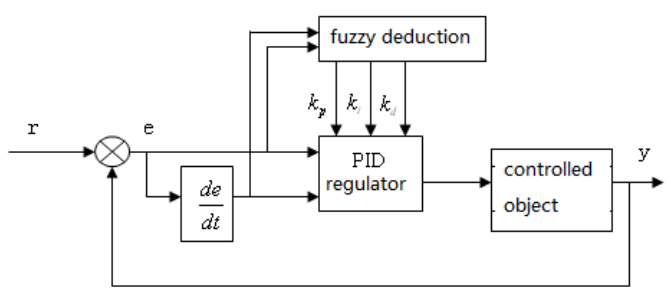

Fig.2. Self-adapting fuzzy PID controller

When using deviation and deviation varying rate to indicate a PID control, the representation is:

$$
u(k)=K_{p} e(k)+K_{i} \sum e(k)+K_{d} e c(k)
$$

Where $u(k)$ is controller output, $e(k)$ is deviation, $e c(k)$ is deviation varying rate, $K_{p}$ is scale coefficient, $K_{i}$ is integral action coefficient, $K_{d}$ is derivative action coefficient.

The calculation formulas that this system achieved self-adjusting PID parameters are as follow:

$$
\begin{aligned}
& K_{p}=K_{p}^{\prime}+\Delta K_{p} \\
& K_{i}=K_{i}^{\prime}+\Delta K_{i} \\
& K_{d}=K_{d}^{\prime}+\Delta K_{d}
\end{aligned}
$$

Where $K_{p}^{\prime}, K_{i}^{\prime}, K_{d}^{\prime}$ are PID initial values, $\Delta K_{p}, \Delta K_{i}, \Delta K_{d}$ are the fuzzy controller's outputs, $K_{p}, K_{i}, K_{d}$ are the final output control parameters.

From the above we knew that self-adapting PID is to find the fuzzy relationships among three parameters $K_{p}, K_{i}, K_{d}$ and e as well as ec, kept checking e and ec during the operation, based on the fuzzy principle to carry out online modification for the three parameters to satisfy different requirements for control parameters with different e and ec so that the controlled object had good dynamical and static characteristics. In the following sections design method for this system controller would be described particularly.

\subsection{The fuzzification of precise value}

The deviation e and deviation varying rate ec of this system are taken as inputs, $K_{p}, K_{i}, K_{d}$ are as three outputs of the fuzzy controller. The domain of the deviation e and deviation varying rate ec is $[-3,3]$, the aggregate in the domain is $\{\mathrm{NB}, \mathrm{NM}, \mathrm{NS}, \mathrm{ZO}, \mathrm{PS}$,
$\mathrm{PM}, \mathrm{PB}\}$, assume the basic domain of $\Delta K_{p}$ is $\{-3,3\}$, the aggregate in the domain is $\{\mathrm{PS} P M \mathrm{~PB}\}$. Assume the basic domain of $\Delta K_{i}$ is $\{-0.1,0.1\}$, the aggregate in the domain is $\{\mathrm{PS} \mathrm{PM} \mathrm{PB}\}$. Assume the basic domain of $K_{d}$ is $\{-1,1\}$, the aggregate in the domain is $\{\mathrm{PS} P M \mathrm{~PB}\}$. See Fig.3 to Fig.7.

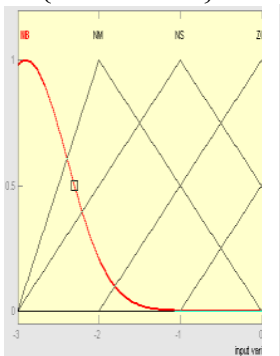

Fig.3

Membership grade of deviation e

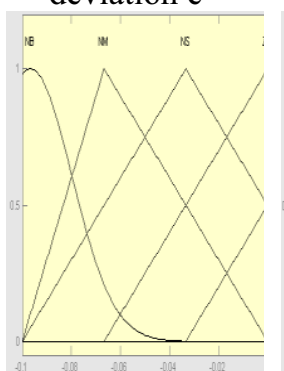

Fig.6 Membership grade of $\Delta K_{i}$

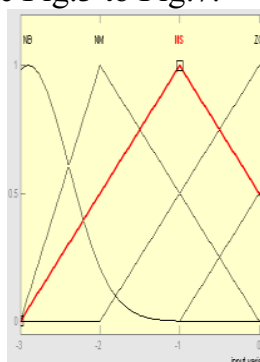

Fig. 4

Membership grade of deviation ec

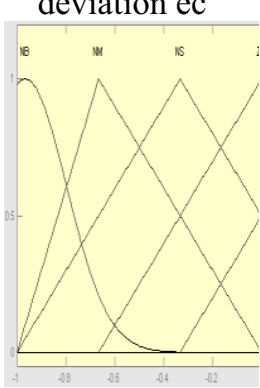

Fig.7

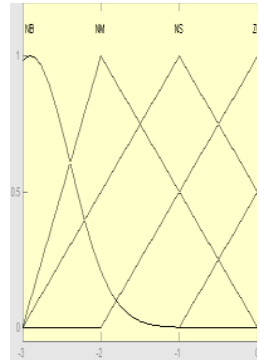

Fig. 5 grade of $\Delta K_{p}$

\subsection{The construction of control regulation}

By a huge amount of experience gathering, there is a kind of non-linear relationship between PID parameter and input controller deviation $\mathrm{e}$ and deviation varying rate ec, the relationship can be described in fuzzy language. Deviation e and deviation varying rate ec have a following relationship with the three parameters of PID controller $K_{p}, K_{i}, K_{d}$. When $|e(t)|$ is larger, to get a faster system responding rate, a larger $K_{p}$ shall be taken to reduce the system time constant and damping coefficient, too large one may lead to a system instability; to avoid the possibility of over-range controlling effect at the beginning of the system, a smaller $K_{d}$ shall be taken to raise the system response. To avoid a bigger overshoot to take out integral action, take $K_{i}=0$. When $|e(t)|$ is right a middle value, a smaller $K_{p}$ shall be taken to get a slightly smaller overshoot; to assure the responding speed, a suitable $K_{d}$ shall be taken, a larger $K_{i}$ can be taken but not too much. When $|e(t)|$ is relatively small, to make sure a good stability, a larger $K_{p}$ and 
$K_{i}$ shall be taken to avoid surge when system is at the equilibrium point, the value of $K_{d}$ shall be proper[4,5]. See Table1 to Table3.

Table 1 The fuzzy regular table of $\Delta K_{p}$

\begin{tabular}{|c|c|c|c|c|c|c|c|}
\hline$K_{p}$ & & & & & \\
& & & & & & \\
& & & & & \\
& & & & & & \\
\hline NB & PB & PB & PM & PM & PS & ZO & ZO \\
\hline NM & PB & PB & PM & PS & PS & ZO & NS \\
\hline NS & PM & PM & PM & PS & ZO & NS & NS \\
\hline ZO & PM & PM & PS & ZO & NS & NM & NM \\
\hline PS & PS & PS & ZO & NS & NS & NM & NM \\
\hline PM & PM & PS & ZO & NS & NM & NM & NB \\
\hline PB & ZO & ZO & NM & NM & NM & NB & NB \\
\hline
\end{tabular}

Table2 Controlling regular table of $\Delta K_{i}$

\begin{tabular}{|c|c|c|c|c|c|c|c|}
\hline$K_{i}$ & $\mathrm{NB}$ & NM & NS & $\mathrm{ZO}$ & PS & PM & PB \\
\hline NB & NB & NB & NM & $\mathrm{NM}$ & NS & $\mathrm{ZO}$ & $\mathrm{ZO}$ \\
\hline $\mathrm{NM}$ & NB & NB & NM & $\mathrm{NS}$ & NS & $\mathrm{ZO}$ & PS \\
\hline NS & $\mathrm{NB}$ & NM & $\mathrm{NM}$ & $\mathrm{NS}$ & $\mathrm{NS}$ & PS & PS \\
\hline $\mathrm{ZO}$ & NM & NM & $\mathrm{NS}$ & NS & $\mathrm{ZO}$ & PS & PM \\
\hline PS & NM & NS & $\mathrm{ZO}$ & $\mathrm{PS}$ & PS & $\mathrm{PM}$ & PM \\
\hline PM & $\mathrm{ZO}$ & $\mathrm{ZO}$ & PS & PS & PM & PB & PB \\
\hline PB & $\mathrm{ZO}$ & $\mathrm{ZO}$ & PS & PM & PB & $\mathrm{PB}$ & PB \\
\hline
\end{tabular}

Table 3 the controlling regular table for $\Delta K_{d}$

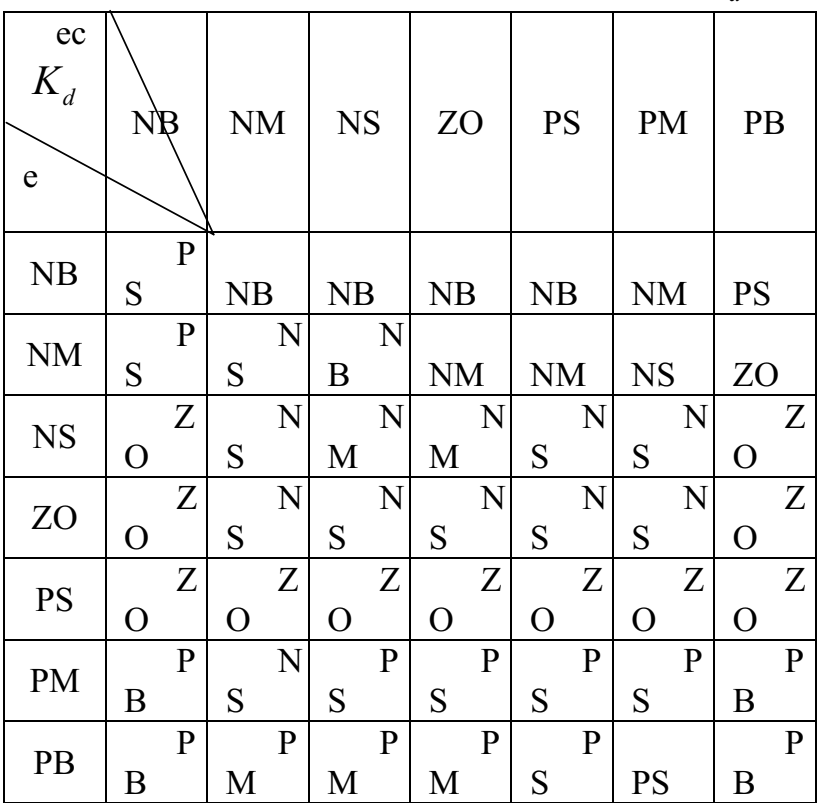

Fuzzy regular table can be written in the form of conditional statement, for $\Delta K_{d}$ the 46 controlling regulars have following forms:

if $\mathrm{e}=\mathrm{NB}$ and $\mathrm{ec}=\mathrm{NB}$ then $\Delta K_{d}=\mathrm{PB}$ if $\mathrm{e}=\mathrm{NB}$ and $\mathrm{ec}=\mathrm{NM}$ then $\Delta K_{d}=\mathrm{PB}$

if $\mathrm{e}=\mathrm{NB}$ and ec=NS then $\Delta K_{d}=\mathrm{PM} \ldots$

In the same way there are also 49 fuzzy regulars for $\Delta K_{i}$ and $\Delta K_{d}$ respectively.

\subsection{Software achievement}

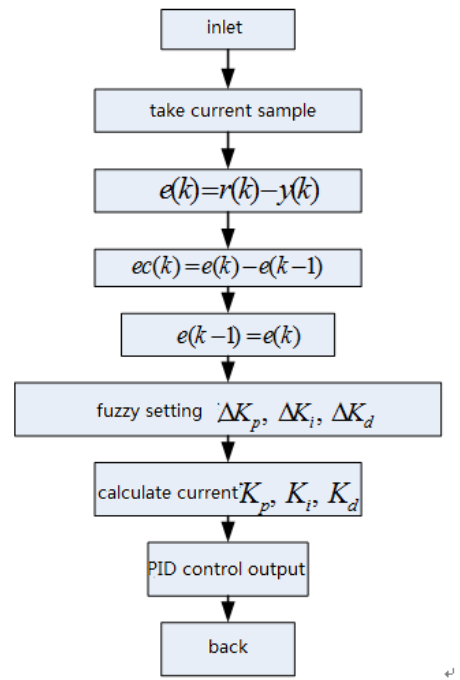

Fig.8. Self-adapting fuzzy PID control program flow chart

In the process of online operation, controlling system accomplish PID parameter online self-adjusting through result solving, table look-up and calculation for fuzzy logic rules. The working flow diagram is shown in Fig.8.

\section{Simulation and analysis self-adapting fuzzy PID control}

Neglecting the system friction and load influence, utilized the control system simplified math model deduced in this article, then carry out system simulation research in the environment of Matlab/Simulink, with two conditions of non-self-adapting fuzzy PID control and applying self-adapting fuzzy PID control method, gave out the respond curves of stepped signal and sine following signal.

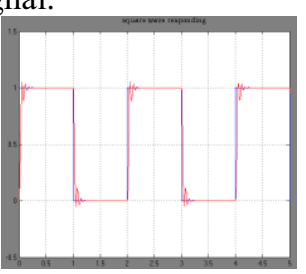

Fig.9. Square wave responding of the system

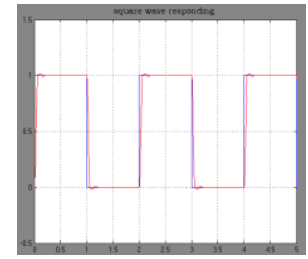

Fig.10. the square wave responding of self-adapting fuzzy PID control system
From Fig.9 it can be seen that the system without modification has a larger overshoot and long adjusting time, the static accuracy is unable to satisfy the technique requirements of control system. After adding up self-adapting fuzzy PID control strategy, as is shown in Fig.10, the system overshoot reduces and 
static accuracy improves, which satisfies the need of system.

\section{Conclusions}

In this article base on the model of wind tunnel motion mechanism control system, the application of self-adapting fuzzy PID control arithmetic on motion mechanism control system is studied, the design method of self-adapting fuzzy PID control arithmetic is properly described, and the simulation model of self-adapting PID control arithmetic is built up in SIMULINK, the parameters of control arithmetic are debugged, and the simulation results are analyzed and compared. According to the analysis, self-adapting fuzzy PID controlling arithmetic is able to achieve a effective action on angle control system, and shows a better control effect and robust.

\section{References}

[1] Chen Taihong. Design of Moving and Measurement Shelf of Single Free Degree and its Control System for Wind Tunnel[J].Industrial Control Computer,2006,19(3) : 1-2.

[2] Chen Taihong. Control System for Transfer Rack of Wind Tunnel[J]. Measurement \&Control Technology, 2005,24(4):80-84.

[3] Song Fengmin. Study on electro-hydraulic servo fuzzy control system of hydraulic cylinder assembling specialized equipment. Master thesis: Shandong University of Technology, 2007.

[4] Zhang Pengcheng. Research on the Intelligent Control Algorithm of 6R Robot. Master thesis: South China University of Technology., 2011.

[5] Mao Bo. Study on self-learning fuzzy control strategy for electro-hydraulic servo system. Master thesis: Yanshan University, 2011. 